\title{
Freak waves - more frequent than rare!
}

\author{
P. C. Liu ${ }^{1}$ and U. F. Pinho ${ }^{2}$ \\ ${ }^{1}$ NOAA Great Lakes Environmental Research Laboratory, Ann Arbor, Michigan, USA \\ ${ }^{2}$ Department of Ocean Engineering, Federal University of Rio de Janeiro, Brazil
}

Received: 21 August 2003 - Revised: 20 November 2003 - Accepted: 8 January 2004 - Published: 8 April 2004

\begin{abstract}
Contrary to the widely held notion that considers the occurrence of freak waves in the ocean as being rare, from an examination of five years of wave measurements made in the South Atlantic Ocean, we found the occurrence of freak waves is actually more frequent than rare.
\end{abstract}

Key words. Meteorology and atmospheric dynamics (waves and tides) - Oceanography: physical (surface waves and tides; air-sea interaction)

\section{Introduction}

A freak wave is a particular type of hazardous ocean wave, that displays a singular wave profile and manifests an extraordinarily large crest or trough with very high local steepness. Apart from a few conjectures and some numerical simulations, we know virtually nothing about this captivating phenomenon. The presumption that freak waves occur rarely seems to be a universally recognized consensus. Yet no known effort has ever been conduced to establish or clarify just what exactly constitutes the notion of being "rare". Here we report that after an examination of five years of wave measurements made in the South Atlantic Ocean, we found the occurrence of freak waves is, in fact, more frequent than rare. This novel finding may not be surprising to the generations of sailors and mariners whose persistent stories of encounters with freak waves had been dismissed as seafaring myths. All the prevailing concepts and many of the currently advanced theoretical approaches on freak waves have been developed primarily based on their rare occurrence (e.g. Janssen, 2003). While there have been persistent doubts as to the existence of freak waves throughout the ages, those doubts have faded away in recent years. Since the well-known time series data of the 1995 New Year's Day freak wave which occurred at the North Sea Draupner platform (Fig. 1) was made public and became widely available (e.g. Trulsen and Dysthe, 1997), it has become the standard characteristic portrait of a freak wave and verifies its existence. It was reported that the platform and some of the equipment were damaged by this freak wave.

Correspondence to: P. C. Liu

(paul.c.liu@noaa.gov)

\section{The finding}

Our study started when we unexpectedly discovered a Draupner-like freak wave case in the Campos Basin (Pinho, 2003), near the northeast coast of Rio de Janeiro, Brazil. The wave was recorded from a heave-pitch-roll buoy moored in over $1000 \mathrm{~m}$ depth. Buoy wave measurement is generally known to underestimate wave crests, and Campos Basin is not a renowned place for freak wave occurrences. Nevertheless, the freak wave recording we stumbled upon in Campos Basin persuaded us to embark on a detailed search through all the available wave measurements there to see if there might be other freak wave cases. The waves were recorded for 17 minutes at $1 \mathrm{~Hz}$ sampling frequency every $3 \mathrm{~h}$ between 1991 and 1995 . Guided by the simple, commonly used criterion of the ratio of maximum wave height to significant wave height greater than 2 for possible freak waves and by further verifing their appearance visually, our search provided us with so many freak wave cases that we were simply astonished. A typical example is shown in Figure 2. We never envisioned that there could be so many cases covering all proportions of significant wave heights that characteristically resemble the Draupner case. We have also found that using zero upcrossing or zero down-crossing approaches in identifying freak wave heights in the time series data do not always lead to the same results. From a total of 7457 data sets recorded in Campos Basin, we found 276 sets contained freak waves that consist of both zero up-crossing and zero down-crossing cases. Figure 3 illustrates their occurrence with respect to time. The data recording periods and freak wave cases are represented in these timeline-type year panels. The horizontal strips represent the time periods of the year that data were recorded. The black + markers that appeared as vertical line segments show the approximate locations in time where freak waves are likely to have occurred. At a sample probability of $3.7 \%$, this is clearly not a picture of rare occurrence. 


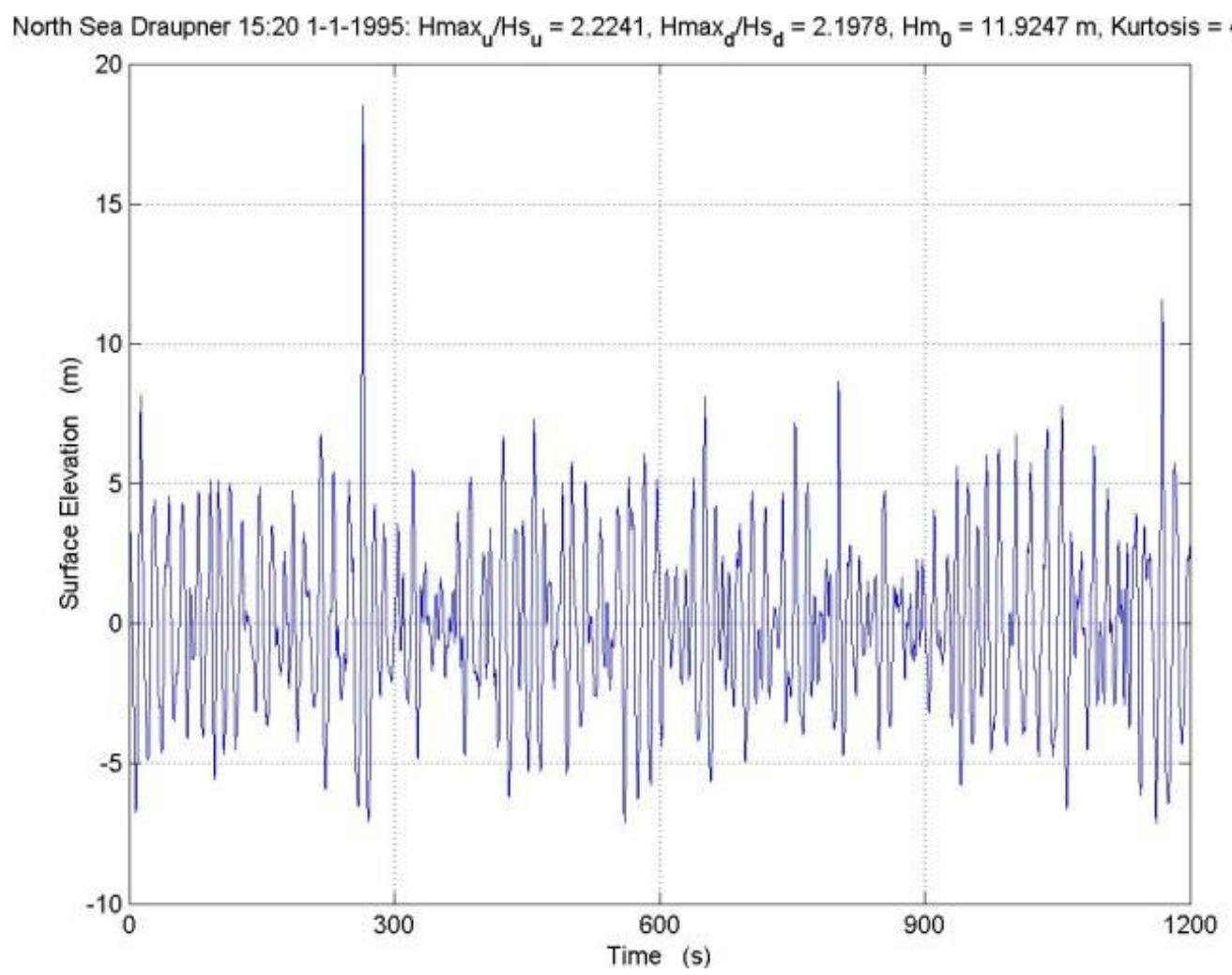

Fig. 1. The time series of a recognized freak wave recorded on 1995 New Year's Day (1995) at the Draupner Platform in the Norwegian sector of the North Sea. The wave elevation was measured by a downward-looking radar system. Data courtesy of Sverre Haver of Statoil.

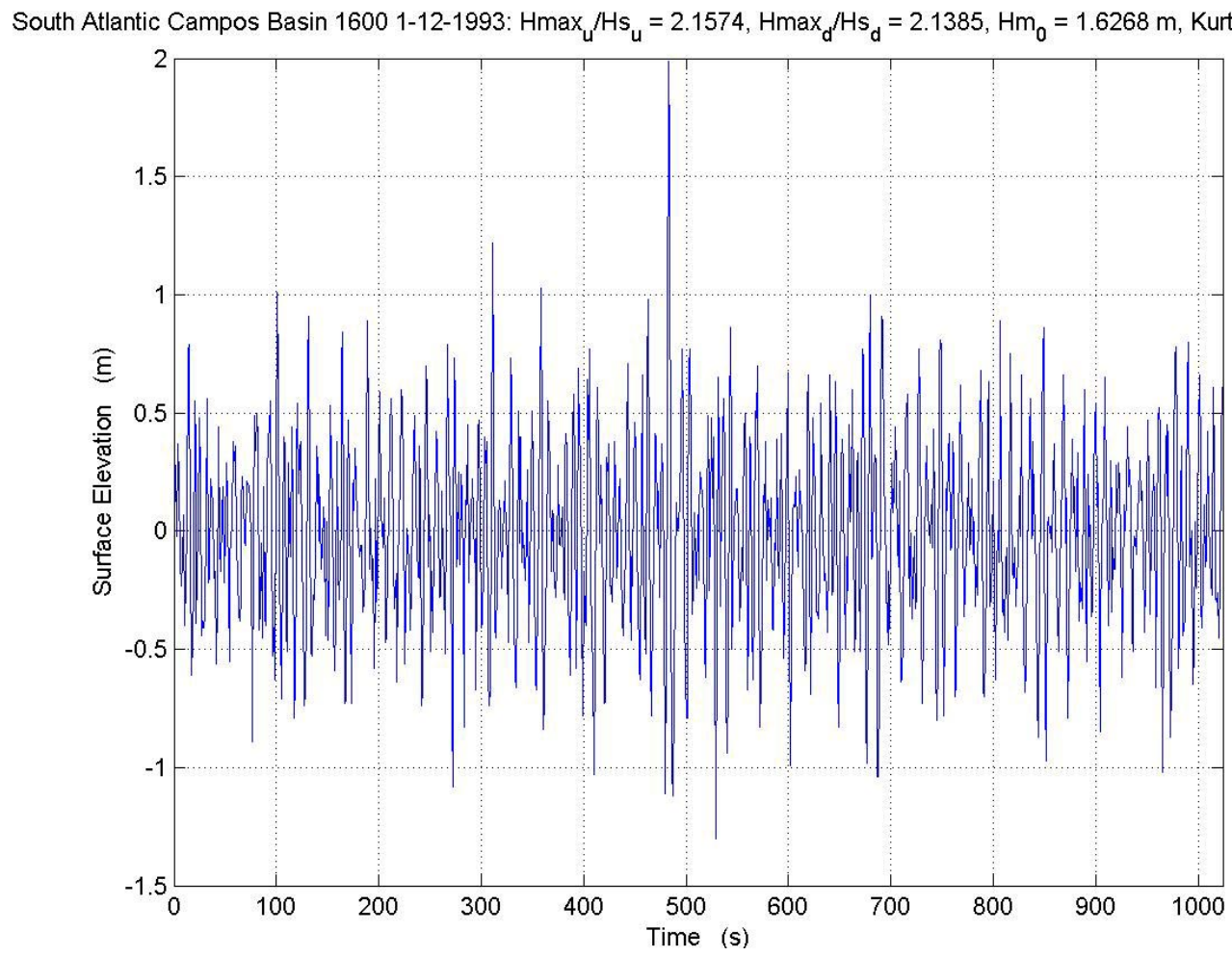

Fig. 2. An example of Draupner-like freak wave time series data recorded in Campos Basin, South Atlantic Ocean, by a heave-pitch-roll buoy moored in over $1000 \mathrm{~m}$ depth. 
P. C. Liu and U. F. Pinho: Freak waves-more frequent than rare!
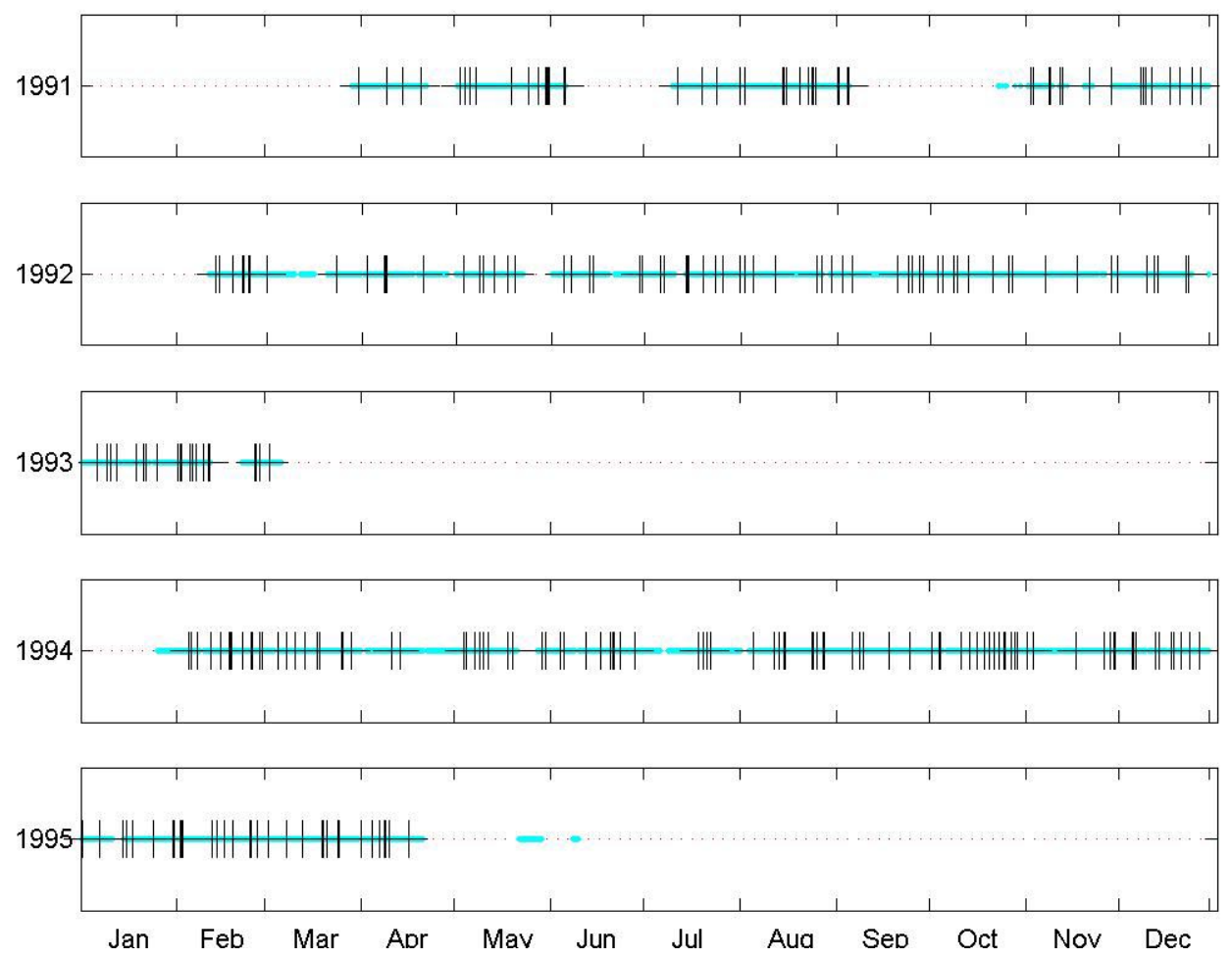

Fig. 3. Incidence of freak wave occurrences in wave data recorded in the Campos Basin of the Southern Atlantic Ocean, which is located in the northeast coast of Rio de Janeiro, Brazil.

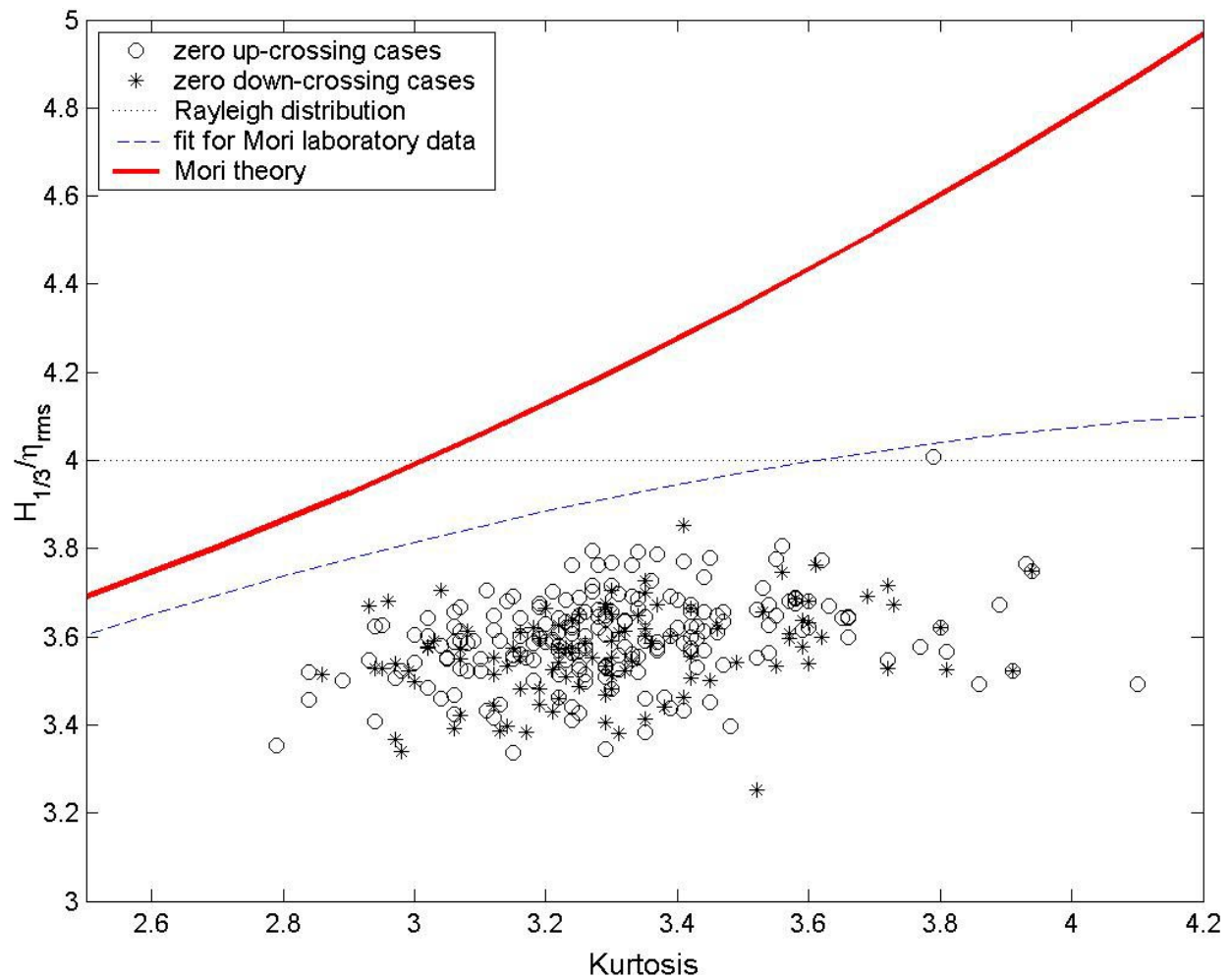

Fig. 4. Correlating $H_{1 / 3} / \eta_{\mathrm{rms}}$ with respect to Kurtosis to illustrate the ineptitude of the Rayleigh distribution as a basis for representing occurrence probability of freak waves. 


\section{An analytical perspective}

Alternatively, Mori (2003) also recognized recently that freak waves appear to be occurring more frequently than what was expected from the Rayleigh distribution and thus developed a modified approach based on a weakly nonGaussian process. Mori formulated a rather complicated transcendental representation that can only be solved numerically. He resourcefully expressed his results in an interesting correlation of the parameters of $H_{1 / 3} / \eta_{\text {rms }}$ versus corresponding Kurtosis, as shown in Fig. 4. We would like to call attention first to the well-known relation of $H_{1 / 3} / \eta_{\mathrm{rms}}=4$, which is effectively the representation of Rayleigh distribution as shown by the thin horizontal dotted line in the figure. Mori's theoretical modification is shown by the thicker solid curve showing a monotonically increasing $H_{1 / 3} / \eta_{\mathrm{rms}}$ with respect to Kurtosis, which adheres to the Rayleigh distribution only at Kurtosis $=3$. To test the theory he conducted a laboratory experiment in a 2-D wave tank with waves generated by the deep water JONSWAP spectrum. The laboratory data is represented by the best fitting dashed line curve below the theoretical curve with a similar but mild trend. Note that JONSWAP spectrum basically reproduces an average ocean condition with one spectral peak, the laboratory results are highly unlikely to be fully realistic. We have proceeded to also plot the Campos Basin data on the same figure as the open circles and asterisks, for zero up-crossing and zero down-crossing cases, respectively, that appeared further below the fitted laboratory curve with the similar and mild trend as well. As Mori indicated, his theoretical result represents an enhancement over Rayleigh distribution in freak wave occurrence probability, conceivably his laboratory results show still more occurrences. We think a plausible interpretation of the results shown in Fig. 4 would be that the natural progression from the Rayleigh distribution through Mori's theoretical modification and laboratory experiment to our oceanic results is qualitatively in the logical track of increasing frequency of the occurrence of freak waves. As our results represent the reality of actual ocean processes, it certainly substantiates our contention that freak waves occur much more frequently than the rareness one usually concluded from the Rayleigh distribution hypothesis.

\section{Concluding remarks}

This simple and rather unexpected result may have a number of broader implications: first, the general perception that freak waves are a rare occurrence should probably be discarded. We have shown that in Campos Basin a singular wave profile that manifests an extraordinarily large crest or trough can happen approximately $3.7 \%$ of the time, and it can happen for significant wave heights of $12 \mathrm{~m}$, or $2 \mathrm{~m}$, or even $0.5 \mathrm{~m}$. Is such an event still considered a freak wave when it occurs at a low significant wave height? It may not be considered a rare occurrence, but it still occurs unexpectedly. Many smaller boats have encountered freak waves in mild wave conditions, some with tragic consequences. Our current conventional wave forecasting and hindcasting systems are based on the premise that the sea state is an ensemble-averaged, stationary Gaussian random process with wave heights following the Rayleigh distribution. Since the Rayleigh distribution is contingent on a stationary process, it basically refutes the existence of freak waves. It requires at least 1655 individual trough-to-crest waves before a maximum wave twice the size of a significant wave height can occur, hence it will never happen in the $1024 \mathrm{~s}$ wave records which we used that averaged only 160 waves in each record. Thus, the conventional system, clearly unequipped for handling nonstationary cases of freak waves that happen more frequently than rarely, accounts more for the kind of waves being called "freak" than for the phenomenon itself. Should we continue to plod along within this framework, or is it timely now to boldly start looking for a new approach and direction for ocean wave studies? Freak waves are intrinsic elements of the ocean wave dynamics. Mariners have at least a $3 \%$ chance of encountering freak waves in their sea-going ventures. This phenomenon should not and need not be treated separately as an isolated, exoteric matter.

Acknowledgement. Topical editor N. Pinardi and the authors thank a referee for his helpful and constructive comments in evaluating this paper. This is GLERL Contribution No. 1303.

\section{References}

Janssen, P. A. E. M.: Nonlinear four-wave interactions and freak waves, J. Phys. Oceanogr., 33, 863-899, 2003.

Mori, N.: Occurrence probability of freak waves in nonlinear wave field, Ocean Engineering, 31, 127-152, 2004.

Trulsen, K. and Dysthe, K.: Freak waves - a three-dimensional wave simulation, Proc. $21^{\text {st }}$ Symposium Naval Hydrodynamics, edited by Wood, E. P., 550-558, 1997.

Pinho, U. F.: Characterization of the sea state in the Campos Basin, M. Sc. Thesis, UFRJ-COPPE, (in Portuguese), 2003. 